\title{
Eine objektive Theorie des guten Lebens
}

Schaber, Peter

DOI: https://doi.org/10.1515/9783110281491.73

Posted at the Zurich Open Repository and Archive, University of Zurich

ZORA URL: https://doi.org/10.5167/uzh-91077

Book Section

Published Version

Originally published at:

Schaber, Peter (2013). Eine objektive Theorie des guten Lebens. In: Hoesch, Matthias; Muders, Sebastian; Rüther, Markus. Glück - Wert - Sinn. Metaethische, ethische und theologische Zugänge zur Frage nach dem guten Leben. Berlin/New York: de Gruyter, 73-88.

DOI: https://doi.org/10.1515/9783110281491.73 


\section{Eine objektive Theorie des guten Lebens}

\subsection{Einleitung}

Verschiedene Arten von Dingen werden als gut für Personen bezeichnet: a) Zustände, in denen sich Personen befinden (,er fühlt sich zur Zeit sehr wohl'); b) Dinge, die ihnen widerfahren (,es ist gut für sie, dass ihr dieser Preis zugesprochen wurde`); c) Phasen ihres Lebens (,er hat eine glückliche Kindheit erlebt') und schliesslich d) das ganze Leben einer Person (,sie hat ein gutes Leben geführt'). So unterschiedlich diese Dinge sind, sie alle werden positiv bewertet, wenn sie gut genannt werden. Was aber heisst es, dass solche Dinge gut sind für Personen? Wann können wir berechtigterweise davon reden, dass etwas gut ist für eine Person? Das ist die Frage, um die es nachfolgend gehen wird. Es soll dabei für eine objektive Theorie des guten Lebens argumentiert werden. Subjektive Theorien des guten Lebens, so die These, erfüllen die Bedingungen nicht, die an eine normative Theorie des guten Lebens zu stellen sind.

Doch wonach fragen wir überhaupt, wenn wir danach fragen, ob etwas gut ist für eine Person? Dass das nicht klar ist, zeigt sich an einer Bemerkung, die der englische Philosoph Bertrand Russell in einem Interview kurz vor seinem Tod gemacht hat. ${ }^{1}$ Auf die Frage eines Journalisten, ob er denn ein gutes Leben geführt habe, antwortete er sinngemäss, das könne man jetzt noch nicht sagen, das hänge davon ab, ob sein Kampf gegen den Nuklearkrieg erfolgreich gewesen sei. Aber kann die Qualität eines Lebens, so fragt man sich, von Fakten abhängig sein, die eine Person selbst nie erleben wird? Hier bedarf es einer begrifflichen Klärung. Kann etwas als gut für eine Person bezeichnet werden, das mit dem, was sie selbst erfährt und ist, nichts zu tun hat? Was kann sinnvollerweise als gut für eine Person betrachtet werden?

Die Schwierigkeit, welche mit Russells Bemerkung verbunden ist, besteht darin, dass das, was gut ist für eine Person, etwas mit ihr zu tun haben sollte. Ob etwas gut oder schlecht ist, muss einen Unterschied für die Person selbst ausmachen. ${ }^{2}$ Es sollte sich dabei um etwas handeln, das - so eine verbreitete Intuition - ihr selbst prinzipiell zugänglich ist. Wenn wir davon ausgehen, dass Russell

1 Vgl. Griffin 1986, 23.

2 Siehe Kagan 1992, 180: „But if something is to make a difference to my level of well-being it must make a difference to me. The facts that constitute my being well-off must be facts about me.“ 
selbst nie erfahren wird, ob das, wofür er sich eingesetzt hat, in Erfüllung gehen wird, kann das auch keinen Einfluss darauf haben, ob sein Leben gut oder schlecht verlaufen ist. Das heisst nicht, dass etwas, was gut ist für eine Person, von ihr selbst eo ipso auch als gut bewertet werden muss. Es muss allerdings von ihr als gut bewertet werden können. Diese positive Bewertung sollte Teil ihres Lebens sein können.

Es wird im Folgenden davon ausgegangen, dass eine Theorie dessen, was es heisst, dass etwas gut ist für eine Person, unter dieser Einschränkung steht. Das, was von der Theorie als gut für Personen identifiziert wird, sollte etwas sein, zu dem die Personen selbst in ihrem Leben prinzipiell Zugang haben.

\subsection{Bewertung und Deliberation}

Bevor wir auf die Frage, was ein für Menschen gutes Leben ist, eingehen, sollten wir klären, welche Rolle eine solche Theorie des guten Lebens im Leben von Personen spielen kann und soll. Es geht genauer um die Klärung dessen, was es für Menschen bedeutet, zu wissen, dass etwas für sie gut oder schlecht ist.

Mit den Ausdrücken ,gut' und ,schlecht' werden generell Dinge bewertet. Bewertungen weisen uns auf Gründe hin. Wenn etwas gut ist für eine Person, dann liefert ihr das Gründe. Wozu aber liefert ihr das Gründe? Die Frage stellt sich, weil hier unterschiedliche Antworten gegeben werden können. Man kann sagen: Dinge, die gut sind für eine Person, liefern ihr Gründe dafür, bestimmte Einstellungen gegenüber diesen Dingen zu haben. Wenn es gut für mich ist, dass ich die Gelegenheit erhalte, ein Buch zum guten Leben zu schreiben, dann sollte ich mich darüber freuen. Und umgekehrt sollte ich über das, was schlecht ist für mich, betrübt sein. Nun ist es naheliegend, im Blick auf diejenigen Dinge, welche gut sind für mich und auf die ich gleichzeitig einen Einfluss haben kann, zugleich als Gründe zum Handeln und Unterlassen zu betrachten. Wenn es gut für mich ist, mehr Sport zu treiben, dann habe ich auch Grund, das zu tun. Die Gründe, die uns das, was gut ist, liefert, können dabei natürlich durch gewichtigere Gründe aufgewogen werden. Es kann sein, dass ich gewichtigere Gründe habe, mich statt dem Sport meiner beruflichen Tätigkeit zu widmen. Das, was gut ist, liefert uns aber in jedem Fall pro tanto-Gründe für Einstellungen wie auch für Handlungen und Unterlassungen.

Die Gründe zum Handeln sind dabei Gründe, die uns, so könnte man sagen, in unserem Nachdenken über unser Tun und Lassen, leiten sollten. Praktische Reflexion ist ein Nachdenken über die Gründe zum Handeln. Hier sollten nicht bloss die Gründe, die darauf beruhen, was gut ist für uns, eine Rolle spielen, sondern auch die Gründe, die uns die Moral liefert. Wenn es aber darum geht, zu be- 
stimmen, was das für mich selbst Beste ist, sollten allein die Gründe, die auf dem persönlichen Gut beruhen, leitend sein.

Letzteres ist allerdings vor dem Hintergrund des berühmten Paradox des Hedonismus umstritten. Nehmen wir - um die Schwierigkeit zu verdeutlichen an, Zustände subjektiven Wohlergehens wären das, was für Personen gut ist, dies die Kernthese einer hedonistischen Theorie des guten Lebens. Das Paradox des Hedonismus besagt: Wir steigern die Chancen, ein in diesem Sinn gutes Leben zu führen, wenn wir uns in der praktischen Reflexion darüber, was wir tun sollten, nicht am subjektiven Wohl, sondern an anderen Dingen orientieren. ${ }^{3}$ Erwartbares subjektives Wohl sollte von uns nicht als Grund zum Handeln gesehen werden. Wer ein gutes Leben führen will, wird andere Dinge als Gründe sehen.

Aber nehmen wir an, subjektives Wohlergehen wäre das, was für Personen gut wäre und eine bestimmte Tätigkeit würde mir solches Wohlergehen versprechen. Wäre das dann für mich kein Grund, die Tätigkeit auszuführen? Wenn es richtig ist, dass die fragliche Tätigkeit gut ist für mich und ich auch weiss, dass dies so ist, dann habe ich einen Grund, entsprechend $\mathrm{zu}$ handeln. Was würde es sonst heissen, dass es gut ist für mich? Es wäre eigenartig zu sagen, $x$ zu tun, wäre gut für mich, aber ich habe keinen Grund, $x$ zu tun. Wenn ich bestreiten würde, einen Grund zu haben, $\mathrm{x}$ zu tun, dann würde ich auch bestreiten, dass das gut für mich ist.

Bedeutet das, dass ich nicht in der Lage bin, ein Leben zu führen, das besser für mich wäre, würde ich mich nicht am subjektiven Wohlergehen orientieren? Das ist zunächst eine empirische Frage, die nicht leicht zu beantworten sein wird. Aber wenn wir annehmen, es wäre so, hiesse das nicht, dass uns subjektives Wohlergehen keine Gründe zum Handeln liefern würde. Es hiesse bloss, dass wir vielleicht besser leben würden, wenn wir diese Konzeption eines guten Lebens für falsch hielten, wenn wir also bestreiten würden, dass sich ein gutes Leben am darin erfahrenen subjektiven Wohlergehen bemisst. Wenn wir diese Theorie aber für richtig halten, sehen wir das subjektive Wohlergehen auch als das, was uns Gründe für unser Tun und Lassen liefert. Dass wir vielleicht insgesamt weniger subjektives Wohlergehen erfahren, wenn wir das tun, ändert nichts daran, dass es das ist, woran wir uns in unserem Handeln orientieren sollten. Dies jedenfalls dann, wenn es sich dabei um die richtige Theorie des guten Lebens handelt. Sie als die richtige Theorie des guten Lebens zu bezeichnen, heisst, subjektives Wohlergehen sowohl als Grund für Einstellungen, als Grund zum Handeln und auch als Grund, sich für gewisse Dinge zu entscheiden, zu sehen.

3 Vgl. Sidgwick 1907, 48: „Here comes into view what we may call the fundamental paradox of Hedonism, that the impulse towards pleasure, if too predominant, defeats its own aim.“ 


\subsection{Subjektive Theorien}

Was heisst es nun aber nun, dass etwas gut ist für Personen? Es gibt eine Fülle von unterschiedlichen Antworten auf diese Frage. Eine wichtige Unterscheidung von Antworttypen, die in der Diskussion eine wichtige Rolle spielen, ist die zwischen subjektiven und objektiven Theorien des guten Lebens. ${ }^{4}$ Und in der Tat lässt sich m. E. an dieser Unterscheidung deutlich machen, worum es in der Debatte um das gute Leben von Menschen zentral geht. Was ist unter einer subjektiven und was unter einer objektiven Theorie des guten Lebens zu verstehen?

Wenden wir uns zunächst der subjektiven Theorie des guten Lebens $\mathrm{zu}$. Subjektive Theorien leben von der Intuition, dass etwas nicht gut sein kann für eine Person, ohne dass die betroffene Person selbst davon etwas mitbekommen würde. ${ }^{5}$ Es kann nicht sein, dass ein Leben gut verläuft und die Person selbst davon nichts merkt oder gar selbst als schlecht einschätzt. Es wäre doch sonderbar, wenn man sagen würde: ,Paul hat ein wunderbares Leben gelebt, obwohl er es selbst für ein sehr schlechtes Leben hielt‘. Sonderbar wäre das nicht zuletzt im Blick auf die oben formulierte Einschränkung, dass das, was gut ist, auch als solches der betroffenen Person zugänglich sein muss.

Nach einer radikalen Version einer subjektiven Theorie des guten Lebens wäre etwas genau dann gut für eine Person, wenn sie den fraglichen Gegenstand selbst als gut bewertet. Damit wird man sich als Vertreter einer subjektiven Theorie allerdings nicht zufriedengeben können. Nehmen wir an, Paul ist überzeugt, dass es für ihn gut ist, wenn er einer Einladung an eine Party folgt. Er wäre allerdings nicht dieser Meinung, wüsste er, was ihn an dieser Party erwartet. Gut - so die entsprechend revidierte Version einer subjektiven Theorie, von der im Folgenden die Rede sein wird - ist etwas für eine Person bloss dann, wenn sie den fraglichen Gegenstand unter der Bedingung für gut hält, dass sie über die relevanten Fakten richtig informiert ist. Die Bewertung darf darüber hinaus auch nicht das Resultat kognitiver Fehler sein. Wenn Paul fälschlicherweise glauben würde, an der Party würde Musik gespielt und er bloss deshalb meint, zur Party zu gehen wäre gut für ihn, beruhte die Bewertung nicht auf mangelnder Information, sondern auf einer falschen Überzeugung. Man könnte entsprechend sagen, dass etwas dann und nur dann gut ist für eine Person, wenn sie (a) den fraglichen Gegenstand für gut hält, und wenn sie (b) die relevanten Informationen besitzt und (c) im Blick auf den Bewertungsgegenstand keine falschen Überzeugungen hat. Etwas ist also nach einer subjektiven Theorie gut für eine Person, wenn sie unter kognitiv vorteilhaften

4 Vgl. Sumner 1996, 27.

5 Vgl. Sumner 1996, 42. 
Bedingungen von der betroffenen Person für gut gehalten wird. Für subjektive Theorien ist die Idee einer Bewertungssouveränität der jeweils betroffenen Person zentral.

Die Bewertungen von Personen kommen in Einstellungen zum Ausdruck. Man hält x für gut und ist entsprechend motiviert, unter den geeigneten Umständen sich für $\mathrm{x}$ zu entscheiden oder sich dafür einzusetzen, dass $\mathrm{x}$ bestehen bleibt. Die Bewertungen sind subjektiven Theorien zufolge mit entsprechenden Handlungsmotivationen verbunden, da sie keine genuinen Überzeugungen darstellen, die richtig oder falsch sein können. Die Bewertung geht der Einstellung nicht voraus. Sie bringt vielmehr eine Einstellung zum Ausdruck. Wir werden auf diesen Punkt in Abschnitt 4.6 zurückkommen. Dabei sind die Bewertungen einer Person genau dann motivational wirksam, wenn die Person rational ist.

\subsection{Objektive Theorien}

Wenden wir uns den objektiven Theorien des guten Lebens zu. Nach objektiven Theorien des guten Lebens können Dinge gut sein für Personen, ohne dass sie von ihnen auch für gut gehalten werden. ${ }^{6}$ Dies gilt diesen Theorien zufolge - und darin unterscheiden sie sich von subjektiven Theorien - auch dann, wenn weder mangelnde Information noch falsche Überzeugungen dafür ausschlaggebend sind, dass jemand etwas für gut hält. So könnte es sein, dass es für Paul gut wäre, zur Party zu gehen, er das aber nicht so sieht, obwohl er weiss, was ihn an der Party erwarten würde. Objektive Theorien gehen davon aus, dass Menschen im Blick auf das gute Leben nicht bloss Opfer von kognitiven Defiziten und Fehlern, sondern auch von genuin evaluativen Fehlern sein können. Paul könnte z. B. fälschlicherweise glauben, dass es schlecht ist, wenn an Partys keine Musik gespielt wird und deshalb nicht hingehen. Eine andere Person könnte fälschlicherweise meinen, die Beziehung mit ihrem Partner sei gut für sie. Dies - so objektive Theorien muss nicht auf kognitive Fehler und Defizite zurückzuführen sein. Es kann auch einfach sein, dass eine Person die falschen evaluativen Überzeugungen hat und sich an ihnen orientiert. Die zentrale These objektiver Theorien lautet entsprechend: Das, was eine Person für gut hält, ist dann für sie gut, wenn sie sich in ihrem Tun und Lassen weder an kognitiven, noch an evaluativen Irrtümer orientiert.

6 Vgl. Sumner 1996, 38: „On an objective theory [...] something can be (directly and immediately) good for me though I do not regard it favourably, and my life can be going well despite my failing to have any positive attitude toward it.“ 
Eine solche objektive Theorie vertritt man beispielsweise dann, wenn man das, was gut ist, an der Idee eines typisch menschlichen Lebens festmacht. ${ }^{7}$ Menschen zeichnen sich, so kann man argumentieren, durch bestimmte Fähigkeiten aus. Diese Fähigkeiten ausüben zu können, ist das, was ein Leben gut macht. Schlecht für Menschen ist entsprechend alles, was sie daran hindert oder was sie selber tun, um sie nicht ausüben zu können. Wer wesentliche menschliche Fähigkeiten nicht ausübt, lebt ein schlechtes Leben, unabhängig davon, ob er selbst es für gut hält oder nicht.

Eine objektive Theorie vertritt man auch, wenn man bestimmte Güter für gut hält, ohne dies an eine Theorie einer typisch menschlichen Lebensform festmachen zu müssen. So kann man sagen, dass glücklich zu sein gut ist für Menschen. Das hat mit Eigenschaften dessen zu tun, was es heisst, glücklich zu sein. Wer weiss, was es heisst glücklich zu sein, sieht auch, dass es gut ist, sich in einem solchen Zustand zu befinden und dass er Gründe hat, nach solchen Zuständen zu streben oder sie, wenn er sich schon darin befindet, zu erhalten. Man kann hier andere Güter wie Freundschaft, beruflichen Erfolg, Autonomie und Wissen hinzunehmen und sagen, dass ein Leben gut verläuft, wenn Menschen Zugang zu diesen Gütern haben. ${ }^{8}$

Objektiven Theorien zufolge sind es die Eigenschaften von Zuständen und Aktivitäten, welche Dinge für uns gut machen. Diese Eigenschaften liegen unabhängig davon vor, ob sie von den betroffenen Personen für gut gehalten werden oder nicht. Genau das ist es, was subjektive Theorien bestreiten. Personen halten nach diesen Theorien Dinge aufgrund von bestimmten Eigenschaften für gut. Aber es sind nicht die Eigenschaften, welche die Dinge gut machen. Zumindest würden sie etwas nicht gut für eine Person machen, wenn sie nicht von der betroffenen Person unter kognitiv vorteilhaften Bedingungen für gut gehalten würde. Objektiven Theorien zufolge kann genau das der Fall sein.

\subsection{Was für objektive Theorien spricht}

So viel zur näheren Bestimmung dessen, was es heisst, eine subjektive und eine objektive Theorie des guten Lebens zu vertreten. Welche Theorie sollte man vorziehen? Eine objektive Theorie des guten Lebens ist genau dann richtig, wenn man falsche evaluative Überzeugungen haben kann, die das eigene Leben betreffen.

7 So z.B. Nussbaum 2006, $74 \mathrm{ff}$.

8 Vgl. Kagan 1992, 170: „Being well-off is simply a matter of one's having the various goods. These might include not only pleasure, but also, for example, friendship, fame, knowledge, or wealth.“ 
Was spricht für diese Auffassung? Zunächst spricht dafür die Weise, wie sich uns diese Dinge in unserer alltäglichen Praxis präsentieren. Wir haben positive Einstellungen gegenüber bestimmten Dingen, weil wir glauben, dass sie gut sind. Und wir glauben auch, Gründe zu haben, uns für gewisse Dinge zu entscheiden, weil und sofern sie gut sind. Den Grund, Freunde zu haben, sehen wir nicht darin, dass wir glauben, das sei gut für uns, sondern darin, dass es für uns gut ist. So jedenfalls stellt sich die Sache aus der Sicht der ersten Person dar. Wir meinen Gründe für Einstellungen zu haben, weil oder sofern die Objekte dieser Einstellungen gut sind. Und wir unterstellen, dass das unabhängig davon der Fall ist, ob wir sie auch für gut halten. Wir können Dinge für gut halten, die nicht gut sind.

Zudem beurteilen wir in unserem Alltag unser eigenes Leben wie auch dasjenige anderer Menschen im Lichte evaluativer Überzeugungen. Weil Gerda von Paul seelisch ausgebeutet wird, halten wir ihre Beziehung zu Paul für schlecht. Gerda bestreitet dies und entsprechend auch, dass die Beziehung zu Paul für sie schlecht sei. Sie könnte auch der Ansicht sein, sie werde zwar ausgebeutet, trotzdem sei die Beziehung für sie gut. Solche Diagnosen müssen an unserer negativen Bewertung nichts ändern. Unsere Bewertung der Beziehung muss dabei nicht davon ausgehen, dass Gerda über relevante Aspekte der Beziehung nicht informiert noch dass sie Opfer falscher nicht-evaluativer Überzeugungen ist.

Gerda bewertet die Fakten falsch. Das ist der Fehler, den sie begeht. Sie sieht nicht, so können wir sagen, dass sie von Paul ausgebeutet wird und die Beziehung entsprechend schlecht für sie ist. Oder sie sieht das zwar, aber sie sieht nicht, dass das ein guter Grund ist, die Beziehung negativ zu bewerten. Genau das ist es, was objektive Theorien behaupten: Es gibt genuine evaluative Irrtümer. Wir können in Bezug auf das, was für uns gut und schlecht ist, falsch liegen, auch wenn wir um alle relevanten Fakten wissen.

\subsection{Persönliche Gründe und evaluative Überzeugungen}

Was lässt sich für subjektive Theorien des guten Lebens vorbringen? Viele meinen, dass es das Scheitern objektiver Theorien des guten Lebens sei, das für subjektive Theorien spricht. Wenn, wie viele meinen, die zentrale These objektiver Theorien falsch ist, bleiben einzig subjektive Theorien als akzeptable Alternativen. Es sind dabei, soweit ich das sehe, zwei Einwände, die sich gegen objektive Theorien des guten Lebens vorbringen lassen.

Zunächst lässt sich bezweifeln - so der erste Einwand -, dass es falsche und wahre evaluative Überzeugungen im Blick darauf geben kann, was für Personen 
selbst gut oder schlecht ist. Wer diesen Einwand vorbringt, muss nicht bestreiten, dass es generell falsche und wahre evaluative Überzeugungen geben kann, sondern bloss, dass es solche geben kann, die sich auf die Qualität des eigenen Lebens bezieht. Die Gründe, auf dem dieser Einwand beruht, haben mit der Subjektrelativität zu tun, von der wir eingangs gesprochen haben.

Man kann aber auch ganz generell bestreiten - so der zweite Einwand - dass es falsche und wahre evaluative Überzeugungen geben kann und so auch die evaluativen Überzeugungen in Frage stellen, die das eigene Leben betreffen. Das ist der grundlegende metaethische Einwand.

Betrachten wir zunächst den ersten Einwand. Wenn etwas gut ist für eine Person, muss es mit der Person etwas zu tun haben. Denn nur dann kann man erklären, dass etwas für diese Person gut ist. Es wäre sonderbar, wenn etwas gut für Paul wäre, Paul davon aber nichts wüsste und er auch davon nichts wüsste, wenn er alle relevanten Fakten kennen würde. Objektive Theorien können dies - und darin liegt das Problem - allerdings nicht ausschliessen. Wenn Pauls evaluative Überzeugungen völlig falsch wären, könnte er ein gutes Leben führen, es selbst aber gleichzeitig für ein sehr schlechtes Leben halten. Subjektive Theorien sind, so kann man argumentieren, im Unterschied zu objektiven Theorien in der Lage, der Idee der Subjektrelativität des guten Lebens Rechnung zu tragen.

Eine subjektive Theorie macht das, was gut ist für Personen an den Einstellungen fest, die sie unter kognitiv idealen Bedingungen haben. Ihre Bewertungen kommen in diesen Einstellungen zum Ausdruck. Es kann so nicht sein, dass das, was gut ist für eine Person, nichts mit ihr zu tun hat. Gut für sie ist das, was ihren Einstellungen entspricht, wenn sie sich über die relevanten Fakten nicht irrt. Damit lässt sich auch dem Faktum Rechnung tragen, dass für verschiedene Menschen unterschiedliche Dinge gut sein können. Während für Gerda die Beziehung mit Paul gut sein könnte, wäre sie für Anna schlecht. Wie die Beziehung für Gerda bzw. für Anna ist, hängt nach einer subjektiven Theorie von den Einstellungen ab, die die beiden jeweils haben, und diese müssen sich nicht entsprechen. So der erste Einwand.

Ist das ein Grund, objektive Theorien zurückzuweisen? Bewertungen kommen in Einstellungen zum Ausdruck. Einstellungen stellen, so die Idee, den Subjektbezug her. Etwas ist gut für Paul heisst: er hat dazu eine positive Einstellung. Es ist allerdings unklar, wieso wir uns auf Einstellungen beziehen müssen, um einen Subjektbezug herzustellen. Man kann zugeben, dass das, was gut ist für Personen, etwas mit dem zu tun haben muss, was Personen sind. Einstellungen sind Eigenschaften von Personen. Was aber privilegiert Einstellungen im Blick auf das, was gut ist für Personen? Diese Frage stellt sich insbesondere deshalb, weil der Subjektbezug über andere Eigenschaften von Personen hergestellt werden kann. Eigenschaften dieser Art sind z.B. Eigenschaften von Personen, die mit ihren 
Fähigkeiten und Begabungen zu tun haben. Dinge können gut sein für Personen, weil sie es ihnen ermöglichen, die dafür erforderlichen Fähigkeiten auszuüben. Das ist z. B. der Grund, wieso wir unmusikalischen Menschen abraten, eine Karriere als Geiger zu verfolgen. Dies tun wir nicht bloss, um die Welt vor kakaphonischen Tönen zu verschonen, sondern auch deshalb, weil wir glauben, dass die betroffene Person damit nicht glücklich werden wird. Und wir würden ihre mögliche Überzeugung, eine Karriere als Geiger wäre gut für sie, für falsch halten.

Subjektive Theorien des guten Lebens könnten dem zustimmen und sagen: Wenn er informiert wäre über das, was es für ihn heisst, eine Geigerkarriere zu verfolgen, würde er nicht mehr motiviert sein, dies zu tun. Aber selbst wenn er es gegen alle Erwartungen des Vertreters der subjektiven Theorie des guten Lebens weiterhin wünschen würde, würden viele seine Überzeugung, es sei gut für ihn, für falsch halten.

Eine von den subjektiven Theorien abweichende Einschätzung dieser Art lässt sich auch gut rechtfertigen. Denn es ist unklar, was Einstellungen in diesem Zusammenhang normativ austragen. Ob eine Karriere als Geiger für die betroffene Person gut ist, hängt von Fakten ab, die unabhängig von seinen Einstellungen der Fall wären: z.B. der Frustration, die er regelmässig erfahren würde, der mangelnden Beachtung, unter der er leiden würde oder auch dem Hohn und Spott, den er möglicherweise über sich ergehen lassen müsste. Einer subjektiven Theorie folgend könnte man sagen: Wenn die betroffene Person um diese Dinge weiss, wird sie die Geigerkarriere auch nicht mehr als für sie erstrebenswert ansehen. Das ist richtig. Allerdings würde sie das genau deshalb nicht mehr tun, weil sie sehen würde, dass aufgrund dieser Fakten eine Geigerkarriere für sie schlecht ist. Ihre Einstellungen würden - wenn alles gut läuft - diesen evaluativen Fakten Rechnung tragen.

Als Vertreter einer objektiven Theorie kann man an der Auffassung, dass das, was gut ist für Personen, etwas mit ihnen zu tun haben muss, festhalten. So kann es sein, dass die Geigerkarriere nicht gut für Paul ist, weil er unmusikalisch ist. Umgekehrt könnte es sein, dass eine Geigerkarriere gut wäre für Anna, weil sie sehr musikalisch ist und sich bei den ersten Versuchen mit der Geige als äusserst talentiert erwiesen hat. Und so wäre es für Anna gut und für Paul schlecht, sich für eine Geigerkarriere zu entscheiden. Das evaluative Faktum beruht in beiden Fällen auf Eigenschaften der betroffenen Personen. In beiden Fällen würden wir das Bestehen des evaluativen Faktums allerdings nicht daran festmachen, dass die betroffenen Personen eine entsprechende Einstellung haben. Man muss m. a. W. nicht auf Einstellungen Bezug nehmen, um der Idee der Subjektrelativität dessen, was gut ist für Personen, Rechnung tragen zu können. 


\subsection{Evaluative Irrtümer?}

Der zweite und allgemeinere Einwand gegen eine objektive Theorie des guten Lebens richtet sich gegen die Idee, es gebe evaluative Irrtümer. Man kann ganz generell bestreiten, dass es evaluative Überzeugungen gibt, die wahr oder falsch sind. Wenn jemand sagt, Geige zu spielen sei gut für ihn, bringt er damit, so kann man argumentieren, nichts anderes als seine positive Einstellung gegenüber der besagten Aktivität zum Ausdruck. Darin kann man sich nicht irren. Einstellungen sind nicht Dinge, die wahr oder falsch sind, sondern vielmehr Dinge, die man hat oder nicht hat. So hat man dem Geigenspiel gegenüber eine bestimmte Einstellung. Man kann zur Kenntnis nehmen, welche Einstellung eine andere Person dem Geigenspiel gegenüber hat. Es ist der Fall, dass eine Person dem Geigenspiel gegenüber eine positive Einstellung hat. Darin irrt sie sich nicht, weil Einstellungen keine Irrtümer sein können. Sie können auf Irrtümern beruhen, aber keine Irrtümer darstellen. So der zweite, allgemeinere Einwand.

Gibt es keine evaluativen Irrtümer? Betrachten wir dazu Folgendes: Paul meint, es sei gut für ihn, Geige zu spielen. Er glaubt das, weil diese Aktivität bestimmte Eigenschaften hat. Das sind die Eigenschaften, die in seiner Begründung, wieso diese Aktivität gut für ihn ist, genannt werden. ,Das Geigenspielen beruhigt mich und lässt mich meine beruflichen Probleme vergessen'. Es sind zwei Arten von Eigenschaften, die hier eine Rolle spielen können: a) nicht-evaluative und b) evaluative Eigenschaften. Erstere werden in rein deskriptiven Begriffen beschrieben; letztere bringen wir mit einem evaluativen Vokabular zur Sprache. Für subjektive Theorien können allerdings bloss nicht-evaluative Eigenschaften eine begründende Rolle spielen. Wenn man sich, wie die subjektive Theorie unterstellt, in Bezug auf die Eigenschaften aufgrund derer man Dinge wählt, irren kann, muss es sich dabei um nicht-evaluative Eigenschaften handeln.

Damit ist aber folgendes Problem verbunden. Zwischen den Eigenschaften und der Bewertung scheint eine normative Beziehung zu bestehen. Es ist jedoch unklar, was nicht-evaluative Eigenschaften bedeutsam macht. Wieso können nicht-evaluative Eigenschaften Gründe liefern? Wieso ist der Umstand, dass das Geigenspiel beruhigt, möglicherweise normativ bedeutsam, der Umstand aber, dass die Geige im Antiquitätenladen gekauft wurde, nicht?

Man könnte sagen, dass es der Umstand ist, dass Paul die Eigenschaften schätzt, die sie normativ bedeutsam machen. Dann sind aber nicht die Eigenschaften normativ bedeutsam, sondern der Umstand, dass sie positiv bewertet werden. Die Eigenschaften lösen bei Paul positive Einstellungen aus. Die Eigenschaften sind nicht die Gründe der positiven Einstellung, sondern das, was die Einstellungen als Reaktion auf die Eigenschaften ermöglicht. Wenn die Eigenschaften vorliegen, dann findet Paul eine positive Einstellung in sich vor. Das ist 
der Mechanismus, den eine subjektive Theorie des guten Lebens beschreibt. Der Zusammenhang zwischen Eigenschaften und Einstellungen wird dann aber nicht normativ verstanden. Er stellt kein Begründungsverhältnis, sondern höchstens einen Kausalzusammenhang dar.

Normativ bedeutsam sind einer subjektiven Theorie zufolge allein die Einstellungen, die bei Personen hervorgerufen werden. Weshalb aber soll der Umstand, dass jemand eine positive Einstellung hat, selbst als normativ bedeutsam betrachtet werden? Dass jemand eine positive Einstellung etwas gegenüber hat, ist ein Faktum in der Welt, das wir zur Kenntnis nehmen können. Wie aber kann das Faktum, dass Paul x für gut hält, als Grund für die Ansicht, dass x für Paul gut ist, gesehen werden? Daraus, dass er x für gut hält, folgt nicht, dass es für ihn gut ist.

Zudem ist dann auch unklar, wieso eine Person - was von allen subjektiven Theorien behauptet wird - allein unter kognitiv vorteilhaften Bedingungen das für gut hält, was gut für sie ist. Nehmen wir an, das Geigenspiel hätte noch eine weitere Eigenschaft, von der Paul aber nichts weiss, z. B. dass der Nachbar über sein Geigenspiel lacht. Die Eigenschaft ist relevant für Pauls Einstellung, weil er sein Geigenspiel nicht mehr in derselben Weise bewerten würde, wüsste er um die Reaktion seines Nachbarn, auf dessen Urteil er sehr viel Wert legt. Aber nehmen wir an, er würde das nie erfahren und sein Geigenspiel nach Massgabe der beiden genannten Eigenschaften bis zum Ende seiner Tage positiv bewerten.

Die Standardversion der subjektiven Theorie würde in einem solchen Fall sagen, dass das Geigenspiel nicht gut ist für Paul. Was aber rechtfertigt eine solche Einschätzung? Klar, Paul würde die Aktivität nicht mehr in der gleichen Weise bewerten, würde er um die anderen Eigenschaften wissen. Auf die Eigenschaften, um die er weiss, reagiert er positiv. Man müsste also sagen: Geige zu spielen, ist für Paul gut, wenn er die beiden Eigenschaften wahrnimmt, nicht gut aber dann, wenn er darüber hinaus auch noch die weitere Eigenschaften wahrnehmen würde. Aber wieso soll das Geigenspiel im ersten Fall auch nicht gut sein für ihn? Was man aus der Perspektive einer subjektiven Theorie sagen kann, ist dies, dass Paul positiv reagiert, wenn er dies weiss und negativ, wenn er noch mehr weiss. Wieso sollten wir die Bewertung davon abhängig machen, dass Paul vollständig über den Gegenstand informiert ist? Was wir sagen können, ist nicht mehr, als dass unterschiedliche Bewertungen bei unterschiedlichem Wissensstand vorliegen. Wieso aber soll eine Bewertung nicht als angemessen angesehen werden, die auf mangelndem Wissen beruht? Eine subjektive Theorie müsste in solchen Fällen sagen: Das Geigenspiel ist dann eben gut für Paul. Bewertungen von Dingen, über die die betroffene Person mangelhaft informiert ist, liessen sich dann nicht kritisieren. Man könnte bloss noch sagen: Das ist die Weise, wie eine Person, die so viel über den Gegenstand weiss, den Gegenstand bewertet. Sie würde den Ge- 
genstand anders bewerten, würde sie auch die andere Eigenschaft kennen. Aber wieso soll dieser Informationsstand den Bewertungsstandard darstellen?

Die subjektive Theorie stellt fest, dass bestimmte Dinge positiv, andere negativ bewertet werden. Damit sagt man aber nichts darüber aus, was gut oder schlecht ist für Personen. Eine Theorie, die das allerdings nicht tut, ist keine normative Theorie des guten Lebens. Sie stellt bloss einen empirischen Zusammenhang fest zwischen Bewertungen und Dingen, die bewertet werden. ,Gegenüber diesen Eigenschaften hat Person x eine positive Einstellung', oder dann in der kontrafaktischen Version: ,Gegenüber diesen Eigenschaften hätte Person x eine positive Einstellung, würde sie sie wahrnehmen'. Es ist aber nicht ersichtlich, inwiefern der konstatierte empirische Zusammenhang zwischen Bewertung und Bewerteten eine normative Bedeutung haben soll. Der Zusammenhang mag jeweils bestehen, er sagt allerdings nichts darüber aus, was gut ist für Personen.

Wenn evaluative Aussagen (,es ist gut für mich, $\mathrm{x}$ zu tun') einfach Einstellungen zum Ausdruck bringen und man an ihnen eine Theorie des guten Lebens festzumachen versucht, ist letzteres nicht mehr als Teil eines normativen Unternehmens zu sehen. Eine Theorie kann uns bloss dann sagen, was gut ist für Personen, wenn sie klarmachen kann, was an den Dingen, die sie für gut hält, normativ bedeutsam ist. Dazu brauchen wir Dinge mit evaluativen Eigenschaften, die uns Gründe für Einstellungen liefern. ,Ich begrüsse es, $\mathrm{x}$ tun zu können, weil x gut ist ${ }^{\star}$. Die Einstellungen, von denen hier die Rede ist, sind nicht bloss Dinge, die wir haben oder nicht haben, sondern vielmehr Dinge, die begründet oder nicht begründet sein können. $\mathrm{Zu}$ sagen, $\mathrm{x}$ ist gut, heisst zu sagen, dass es Gründe gibt, die für $\mathrm{x}$ sprechen. Und entsprechend gilt, dass wenn $\mathrm{x}$ gut ist, es solche Gründe gibt. Diese Gründe müssen durch die Eigenschaften geliefert werden, denen gegenüber man eine positive Einstellung hat. ${ }^{9}$

Wenn das richtig ist, dann bringt man mit evaluativen Aussagen Dinge zum Ausdruck, die bestehen oder nicht bestehen. Die Gründe, die dabei geltend gemacht werden, sprechen für ihr Bestehen. Es kann sein, dass nichts für ihr Bestehen spricht. Wer trotzdem meint, der fragliche evaluative Sachverhalt bestünde, irrt sich. Er wäre dann das Opfer eines evaluativen Irrtums.

9 Dabei kann es sein, dass eine Person Gründe hat, $x$ zu tun und zugleich auch Gründe, y zu tun, ohne dass sich klar sagen liesse, für welche Option die stärkeren Gründe sprechen. Die Gründe könnten ungefähr gleich stark sein. Oder sie könnten inkommensurabel sein. In beiden Fällen müsste man sich einfach entscheiden. Die evaluativen Überzeugungen ,Es ist gut für mich, $x$ zu tun' und ,Es ist gut für mich, y zu tun “ wären beide richtig. Dasselbe würde in dem Fall für die Aussage ,Es ist richtig, einer der beiden Optionen zu wählen` gelten; vgl. dazu Raz 1986, $321 \mathrm{ff}$. 


\subsection{Was für uns gut ist}

Wie wir oben gesagt haben, gibt es unterschiedliche Varianten objektiver Theorien des guten Lebens. Eine Variante macht das, was gut ist für Menschen, an einer typisch menschlichen Lebensform fest, eine andere an intrinsisch wertvollen Gütern, die zu wählen für Menschen gut ist. Allgemein lässt sich dabei folgendes festhalten: Gut ist nach einer objektiven Theorie etwas für eine Person dann, wenn der fragliche Gegenstand evaluative Eigenschaften hat, die der betroffenen Person einen Grund liefern, ihn zu wählen, hervorzubringen oder zu bewahren. Ob solche Gründe vorliegen, hängt nicht von den Einstellungen der Person ab. Eine Person kann Dinge tun, die für sie schlecht sind, weil sie evaluative Überzeugungen hat, die sich auf ihr Leben beziehen und falsch sind.

Betrachten wir dazu ein Beispiel: Ist es für Paul gut, an die Party seines besten Freundes zu gehen? Man kann sagen: Das ist genau dann der Fall, wenn der Partybesuch evaluative Eigenschaften besitzt, die Paul nahelegen, zur Party zu gehen. So könnte gelten: Paul wird an der Party anregende Gespräche führen können; er wird ihm bekannte Personen treffen können, mit denen er sich bestens amüsieren wird etc. Das sind mögliche Gründe für Paul, zur Party zu gehen. Ihre Beschreibung ist nicht rein deskriptiver Natur. Sie enthalten vielmehr evaluative Begriffe wie ,anregend“ und ,bestens amüsieren'. Der Partybesuch hat Eigenschaften, die Paul Gründe liefern: Die Gespräche werden für ihn anregend sein; er wird sich mit ihnen bestens amüsieren können. Auf seinen Kollegen, der auch eingeladen wurde, trifft all dies möglicherweise nicht zu, und entsprechend hätte er auch keinen Grund, zur Party zu gehen. Was gut ist für Paul, kann schlecht sein für Thomas. Das hat nichts mit ihren Bewertungen, sondern vielmehr damit zu tun, welche evaluative Beschreibung des Anlasses aus ihrer Sicht jeweils wahr wäre. In Pauls Beschreibung könnten Begriffe wie ,anregend' ,höchst amüsant', in Thomas’ Beschreibung, ödes Philosophieren' und ,schrecklich langweilige Gesprächspartner‘ angemessen sein. Das hat mit Fakten zu tun, welche die beiden Personen betreffen: dem Faktum z.B., dass Paul sich intensiv mit bestimmten Fragen philosophischer Natur beschäftigt und Thomas dies nicht tut. Die Unterschiede haben - allgemein gesprochen - mit dem zu tun, was die Personen jeweils sind und tun. So hat Paul einen Grund, zur Party zu gehen und Thomas eben nicht.

Eine in diesem Sinne objektive Theorie des guten Lebens hat auch keine Schwierigkeiten, individuelle Unterschiede im Blick auf das, was für Menschen gut oder schlecht ist, zu erklären. Sie kann der Idee der Subjektrelativität des Guten Rechnung tragen. Das, was gut ist für Personen, ist dem, was sie sind, nicht fremd. Dinge sind gut für sie, weil sie bestimmte Eigenschaften besitzen, weil sie gewisse Talente und Fähigkeiten haben; weil sie bestimmte Projekte verfolgen; weil ihnen bestimmte Dinge wichtig sind. Letzteres ist nicht mit einer subjektiven Bewertung 
zu verwechseln. Dinge sind Personen wichtig aufgrund dessen, was sie sind und auch aufgrund dessen, was sie tun und getan haben. Es kann für mich wichtig sein, ein Buch zum guten Leben, an dem ich lange gearbeitet habe, zum Abschuss bringen zu können. Es würde mir ein Gefühl tiefer Befriedigung geben, wenn mir das gelingen würde. Das wäre auch dann der Fall, wenn ich mir möglicherweise sagen würde, dass das eh nicht wichtig sei und ich mich ja eh mit Theorien des guten Lebens gar nicht hätte beschäftigen sollen. Die Eigenschaften von Personen bilden den Hintergrund, vor dem bestimmte Dinge für Personen evaluative Eigenschaften haben, die ihnen Gründe liefern, sie zu wählen, ihre Existenz zu begrüssen, über ihren Besitz sich zu freuen usw. Das, was objektiv gut ist für Personen, ist gut für einzelne Personen, nicht einfach gut.

Eine objektive Theorie des Guten kann nicht bloss der Subjektrelativität Rechnung tragen, sie vermag auch - anders als subjektive Theorien - zu erklären, was es heisst, dass etwas gut für eine Person ist. Subjektive Theorien stellen fest, dass das Vorliegen bestimmter Eigenschaften zu positiven oder negativen Bewertungen führen oder mit positiven oder negativen Bewertungen korrelieren. Objektive Theorien demgegenüber sagen, dass evaluative Eigenschaften auf dem Hintergrund dessen, was Personen sind, Personen Gründe liefern, sie für gut zu

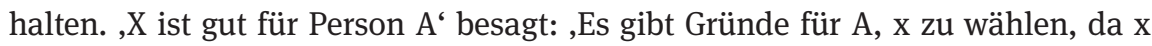
bestimmte evaluative Eigenschaften besitzt'. Das heisst es, den Subjektbezug eines ,gut sein für' verständlich zu machen. Allein objektive Theorien des guten Lebens sind dazu in der Lage. Und das spricht für sie.

Nun kann man einwenden, dass man die Subjektrelativität des Guten noch nicht erklärt hat, wenn man ,x ist gut für $\mathrm{A}^{\text {‘ }}$ als ,Es gibt Gründe für $\mathrm{A}, \mathrm{x}$ zu tun versteht. ${ }^{10}$ Wenn beispielsweise gilt, dass A moralisch verpflichtet ist, andere nicht physisch zu verletzen, dann hat er Gründe, so zu handeln. Diese Gründe sind nicht subjektrelativ, obwohl sie Gründe für A sind. Es sind nicht Gründe, die auf besonderen Eigenschaften A's beruhen.

Es ist richtig, dass prudentielle im Unterschied zu moralischen Gründen subjektrelativ sind. Ich habe im prudentiellen Sinn einen Grund, x zu tun, wenn x für mich eine evaluative Eigenschaft hat, die sie für andere nicht auch haben muss. Für Paul hat der Partybesuch evaluative Eigenschaften, die ihm Gründe liefern, zur Party zu gehen, die der Partybesuch für Thomas nicht besitzt. Das hat mit Eigenschaften der betroffenen Personen zu tun. Paul ist jemand, dem Tanzen Spass bereitet, Thomas nicht. Prudentielle Gründe sind Gründe für Personen, die auf den besonderen Eigenschaften von Personen beruhen. Das macht sie zu

10 Ich verdanke diesen Einwand Christoph Halbig. 
subjektrelativen Gründen. Sie sind gleichzeitig objektive Gründe, weil sie nicht von den Einstellungen von Personen, sondern von dem, was sie sind, abhängig sind.

\subsection{Wie es sich anfühlt}

Objektive Theorien schliessen nicht aus, dass Menschen sich über das, was gut für sie ist, irren können. Wer sich in seinem Leben an falschen evaluativen Überzeugungen orientiert, lebt kein gutes Leben. Es stellt sich jedoch die Frage, wie unabhängig das Faktum, dass etwas gut ist für eine Person, von der Weise, wie es von der betroffenen Person erfahren wird, sein kann. ${ }^{11}$ Ronald Dworkin schreibt:

(S)omeone who leads a boring, conventional life without close friendships or challenges or achievements, marking time to his grave, has not had a good life, even if he thinks he has and even if he has thoroughly enjoyed the life he has had. ${ }^{12}$

Nach Dworkin kann ein Leben schlecht sein, auch wenn es von der betroffenen Person als zutiefst befriedigend erfahren wird. Er vertritt, was man als einen starken Objektivismus bezeichnen könnte. Ein schwacher Objektivist würde dagegen sagen: Ein Leben kann nicht schlecht sein, wenn es dauerhaft als befriedigend erfahren wird. Der Umstand, dass man etwas als zutiefst befriedigend erfährt, zeigt, dass die Dinge, die für diese Erfahrung verantwortlich sind, für die betroffene Person positive evaluative Eigenschaften hätten. Umgekehrt gilt: Wäre das dauerhaft nicht der Fall, würden sich diese evaluativen Eigenschaften für die betroffene Person nicht realisieren. Sie wäre dann eben nicht so beschaffen, dass für sie diese Dinge positive evaluative Eigenschaften haben.

Der Vorteil eines schwachen Objektivismus besteht darin, dass er das, was im prudentiellen Sinn gut ist für Personen, besser als ein starker Objektivismus an das zu binden vermag, was Personen sind. Es ist unklar, wie ein starker Objektivismus der Idee der Subjektrelativität Rechnung zu tragen vermag. Wenn etwas, wie Dworkin meint, schlecht sein kann für eine Person, obwohl sie es dauerhaft als befriedigend erfährt, dann hat das gute Leben nichts mit dem, was Personen sind, zu tun. Ein konventionelles Leben, um ein Beispiel von Dworkin zu nehmen, ist dann schlecht für A, weil ein solches Leben eben schlecht ist. Das jedenfalls scheint die Überlegung eines starken Objektivisten zu sein. Wenn man aber die Idee ernst nimmt, dass Dinge für die einen im prudentiellen Sinn gut und

11 Christoph Halbig hat mich auf diesen Punkt hingewiesen.

12 Dworkin 2011, 42. 
gleichzeitig für andere schlecht sein können, erweist sich ein schwacher Objektivismus als die bedeutend plausiblere Theorie.

Man kann einer objektiven Theorie zufolge das falsche Leben führen. Das aber bedeutet nicht, dass das, was gut ist für Personen, nichts mit ihnen zu tun hätte. Menschen können sich über das, was für sie gut ist, irren, weil sie dem nicht Rechnung tragen können, was sie selbst sind und sein können. Das sind Dinge, zu denen sie selbst prinzipiell Zugang haben. Es sind nämlich Eigenschaften ihrer Person. Ungeachtet dessen muss das, was Menschen faktisch für gut halten, nicht das sein, was gut für sie ist. Diese Möglichkeit, sich im Blick darauf, was für die eigene Person gut ist, irren zu können, ist auch das, was die Frage danach, was denn ein gutes Leben sei, motiviert. Diese Frage ist nämlich nicht die Frage danach, was wir für gut halten (das wissen wir), sondern die Frage, was wir für gut halten sollen.

\section{Literatur}

Dworkin (2011): Ronald Dworkin, „What Is A Good Life?“, New York Review of Books 59, 41- 43. Griffin (1986): James Griffin, Well-Being: Its Meaning, Measurement, and Moral Importance, Oxford.

Kagan (1992): Shelley Kagan, „The Limits of Well-Being“, Social Philosophy and Policy 9 , $169-189$.

Nussbaum (2006): Martha Nussbaum, Frontiers of Justice, Cambridge, MA.

Raz (1986): Joseph Raz, The Morality of Freedom, Oxford.

Sidgwick (1907): Henry Sidgwick, The Methods of Ethics, London.

Sumner (1996): Leonard W. Sumner, Welfare, Ethics \& Happiness, Oxford. 